\title{
The Role of AMPAR Trafficking Mediated by Neuronal Pentraxins in Cocaine-induced Neuroadaptations
}

\author{
Alejandra M. Pacchioni and Peter W. Kalivas \\ Department of Neurosciences, Medical University of South Carolina, Charleston, South Carolina
}

PharmSight on Pacchioni AM, et al. Neuronal pentraxins modulate cocaine-induced neuroadaptations. J Pharmacol Exp Ther 2009;328:183-92.

\begin{abstract}
Glutamate AMPA receptors (AMPAR) in the nucleus accumbens have an important role in cocaine-induced neuroadaptations. Neuronal pentraxins function in the extracellular matrix to bind AMPAR. Three neuronal pentraxins have been described Narp, NP1 and NPR. Narp and NP1 cluster AMPA receptors, while NPR contributes to removing AMPA receptors during mGluRdependent long-term depression. We recently demonstrated that each pentraxin contributes to cocaine-induced neuroadaptations in a way that is consistent with its role in AMPAR clustering and trafficking. Thus, Narp and NP1 deletion promoted cocaine-induced place preference and showed blunted AMPA induced locomotion after cocaine withdrawal. In contrast NPR deletion augmented the AMPA response and was without effect on place preference. Consistent with reduced AMPA responsiveness after chronic cocaine in Narp KO mice, GluR1 was reduced in the postsynaptic density (PSD) fraction of Narp KO mice withdrawn from cocaine. These findings will be discussed in light of recent data showing that rats withdrawn from cocaine have marked deficits in developing long-term potentiation and long-term depression.
\end{abstract}

Keywords: Neuronal pentraxins; AMPAR; mGluR5induced LTD; Cocaine

Chronic cocaine exposure induces changes in glutamate neurotransmission in nucleus accumbens (NAcc) that are linked to behavioral sensitization and addiction (1). For example, withdrawal from chronic cocaine increases the expression of the

Received 03/19/09; accepted 05/06/09

Correspondence: Dr. Alejandra M. Pacchioni, Department of Neurosciences, Medical University of South Carolina, 173 Ashley Avenue, BSB 403, Charleston, SC 29425, USA. Tel. 843-792-4400, Fax. 843-792-4423. email: pacchio@musc.edu
GluR1 subunit of AMPA receptors (AMPAR) in accumbens $(2,3)$, and correspondingly increases the ratio of AMPA to NMDA currents in accumbens medium spiny neurons (4). Also, AMPA injected into the NAcc demonstrates behavioral cross sensitization with cocaine and induces cocaineseeking behavior in cocaine-experienced rats, while AMPA antagonists block cocaine-induced behavioral sensitization and seeking behavior (5-7). Moreover withdrawal from chronic cocaine reduces extracellular glutamate due to a downregulation of the cystine-glutamate antiporter in the NAcc (8), which reduces glutamatergic tone on the group II presynaptic glutamate metabotropic receptors (mGluR2/3) (9), and results in an increase of glutamate release during the reinstatement of cocaine seeking (10).

At the excitatory synapses, AMPAR are aggregated by proteins in both the intracellular compartment and the extracellular matrix. Neuronal Pentraxins (NPs) are among the proteins enriched in the extracellular matrix $(11,12)$. Three different NPs have been described: Narp (neuronal activity regulated pentraxin, also termed NP2), NP1 (neuronal pentraxin 1) and NPR (neuronal pentraxin receptor) (13). Narp is an immediate early gene that is induced by synaptic activity, while NP1 and NPR are constitutively expressed (14). Narp and NP1 multimerize, thereby enhancing the clustering and synaptogenic activity of the individual proteins (14). In contrast to Narp and NP1, which are secreted proteins, NPR possesses a single transmembrane domain. Recently, Cho and colleagues reported an association between NPs and group I metabotropic receptors (mGluR1/5) induced long-term depression (LTD) (15). Thus, NPs appear to have opposing effects on AMPAR aggregation at synapses, with Narp and NP1 acting to enhance 
AMPAR clustering and NPR acting to remove AMPAR (Fig.1).

Consistent with a shared AMPAR clustering function for Narp and NP1, we recently reported that deletion of either NP caused similar alterations in spontaneous and cocaine-induced behavior, and blunted motor response elicited by stimulating AMPAR in the NAcc during cocaine withdrawal. On the other hand, NPR deletion potentiated the motor effect of AMPA. We also showed that GluR1 subunit of AMPAR is down-regulated in the postsynaptic density (PSD) after abstinence from chronic cocaine in Narp KO mice (16). As reported previously, withdrawal from chronic cocaine potentiates the motor stimulant effect of intra-accumbens AMPA (6), and the fact that this potentiation was inhibited in Narp KO is consistent with the marked downregulation of GluR1 in the Nacc PSD in Narp KO mice withdrawn from chronic cocaine administration (16). Taken together, our findings suggest opposing actions of Narp and NP1 versus NPR in cocaineinduced plasticity that parallel their differential actions in synaptogenesis and excitatory transmission. Narp and NP1 are able to multimerize and promote clustering of AMPAR $(11,14)$, while NPR is thought to bind to the Narp/NP1/AMPA clusters and promote internalization of the clustered receptors (15). Our data also implicate the NPs as potential regulators of the behavioral and cellular adaptations produced by chronic cocaine.

We also measured cocaine rewarding properties after individual deletion of each pentraxin using conditioned place preference. Once more, Narp and NP1 KO mice showed similar results where cocaine place preference was enhanced and NPR KO showed no effect on cocaine place preference compared to their WT. Moreover, Narp and NP1 KO showed a delayed expression of sensitization, and NPR was similar to its WT. Considering these results together may seem paradoxical since locomotor sensitization and rewarding properties usually go together with an increase locomotor response to AMPAR stimulation. However, by removing the pentraxins we produce changes in the fine tuning of glutamate synapses which may differentially affect cocaineinduced behavioral responses. Recently, Bachtell et al (17) showed the overexpression of GluR1 holding a single point mutation that reduces synaptic current through AMPAR decreases AMPA induced locomotion after a cocaine treatment, but increases cocaine sensitization and reinstatement. Along these same lines, Narp and NP1 KO showed responses akin to the over-expression of the mutant GluR1, suggesting that in the absence of Narp or NP1 the function of AMPAR may be decreased; which is also suggested by the lower DHPG induced locomotor activity in Narp KO and by the reduced AMPA induced activity in Narp/NP1 KO shown in our study (16). Previous studies have shown that the activation of group I mGluR by DHPG in the NAcc of naïve animals produces an increase in locomotor activity that is mediated by AMPAR (18).

As mentioned above we found a reduction in GluR1 in the PSD subfraction from the ventral striatum of Narp KO after withdrawal from cocaine that reveals an interesting interaction between cocaine regulation of AMPAR and Narp (16). Previous studies showed an increase in GluR1 after withdrawal from chronic cocaine that was associated with the development of behavioral sensitization (3). Given the lack of change in GluR 1 in the non-PSD fraction (16), we proposed that the reduced capacity to cluster AMPAR after chronic cocaine in Narp KO mice resulted from a change in the trafficking of GluR1 into or out of the PSD subfraction, rather than a change in synthesis of AMPAR subunits.

It is tempting to speculate that the functional segregation of NPs in synaptogenesis and excitatory transmission may underlie the differential effects of individual NP gene deletion on AMPA-induced motor activity, cocaine-induced place preference and behavioral sensitization. Thus, the presumed deficit in AMPAR clustering associated with Narp or NP1 gene deletion would be predicted to impair AMPAR signaling. In fact, Narp and NP1 deletions inhibited AMPA-induced locomotion. Conversely, NPR KO mice show reduced mGluR5-dependent LTD due to reduced internalization of AMPAR in NPR KO (15), which would be predicted to potentiate AMPAinduced responses. Correspondingly, we demonstrated that NPR deletion augmented the motor response to AMPA administration into NAcc(16).

Recently, we found that cocaine selfadministration induces metaplasticity that impairs the ability of prefrontal cortex to produce long term potentiation (LTP) or LTD in NAcc, and that Nacetylcysteine (NAC) a drug that inhibits cocaine reinstatement by restoring glutamate levels in the NAcc also reverses cocaine metaplasticity (19). Interestingly, the restoration of LTP was linked to NAC-induced restoration of the glutamate tone on presynaptic group II metabotropic receptors (mGluR 2/3) while the restoration of LTD was linked to restoration of the glutamate tone on the postsynaptic mGluR5. However, while the 


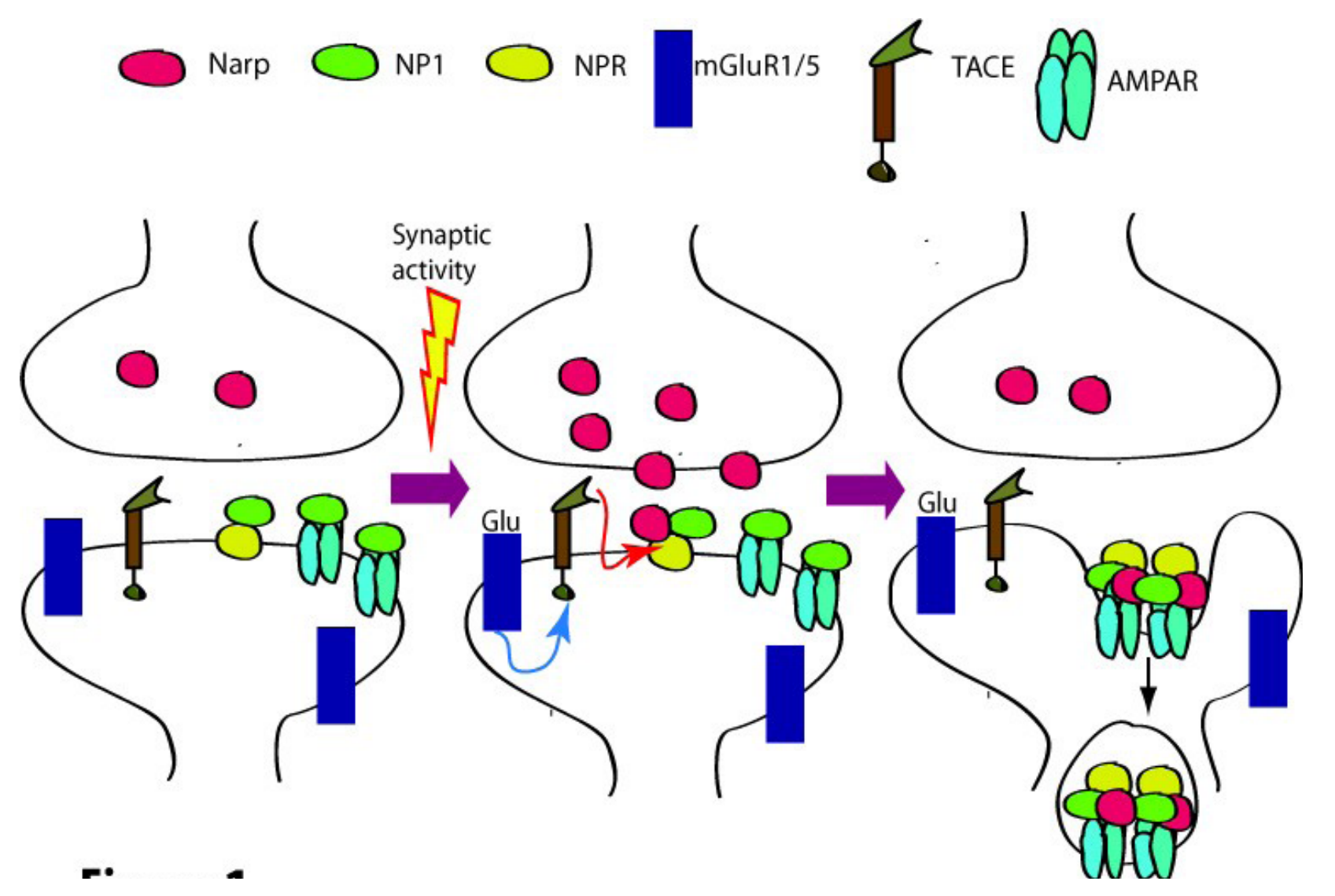

Figure 1. Model for Pentraxin function. Synaptic activity induces the release of Narp, and the activation of TACE through the stimulation of mGluR5 receptors. TACE cleaves NPR's transmenbrane domain releasing NPR, which then clusters Narp-NP1-AMPAR inducing its endocytosis. Figure adapted from Cho et al (15).

pharmacological stimulation of mGluR2/3 prevents cocaine seeking $(20,21)$, the stimulation of mGluR5 with a positive allosteric modulator in the presence of a lower dose of NAC promotes cocaine seeking reversing NAC effects (19). These data suggest that the impairment in mGluR5-induced LTD may be protective and limit the intensity of cocaine seeking (19).

Cho and colleagues recently demonstrated that NPR participates in mGLuR1/5 induced LTD (15). The authors showed that the stimulation of mGluR1/5 activates the extracellular metalloprotease ADAM 17 (TACE) which cleaves and releases NPR to promote endosome-dependent removal of AMPAR from the synapse. Thus, NPR KO mice showed a reduced mGluR5-induced LTD (15) and consistent with a protective role of impaired mGluR5-induced LTD, we reported that NPR KO mice have no effect on cocaine rewarding properties and no differences in locomotor sensitization compared to WT, in spite of an augmented behavioral response to AMPAR stimulation (18).
Furthermore, previous work from the lab also showed that mGluR5 protein levels are reduced in NAcc in rats withdrawn from cocaine, also implying that mGluR5 is functionally impaired (18). Taking all these data together leads us to suggest one possible mechanism that links mGluR5 LTD impairment and NPR as part of cocaine neuroadaptations. In other words, mGluR5 downregulation after cocaine withdrawal would impair TACE activation, probably reducing NPR release and AMPAR removal from the surface, leading to an impairment in mGluR5-induced LTD. Certainly further experiments need to examine this mechanism (Fig. 2).

The function of the other two pentraxins, Narp and NP1, is to cluster the AMPAR from the extracellular matrix (14). Our results suggest that deficits in pentraxin-induced clustering of the AMPAR may decrease receptor function (i.e. blunted response to AMPA stimulation in Narp and NP1KO, and blunted response to DHPG in Narp KO) which seems to favour cocaine-induced neuroadaptations 


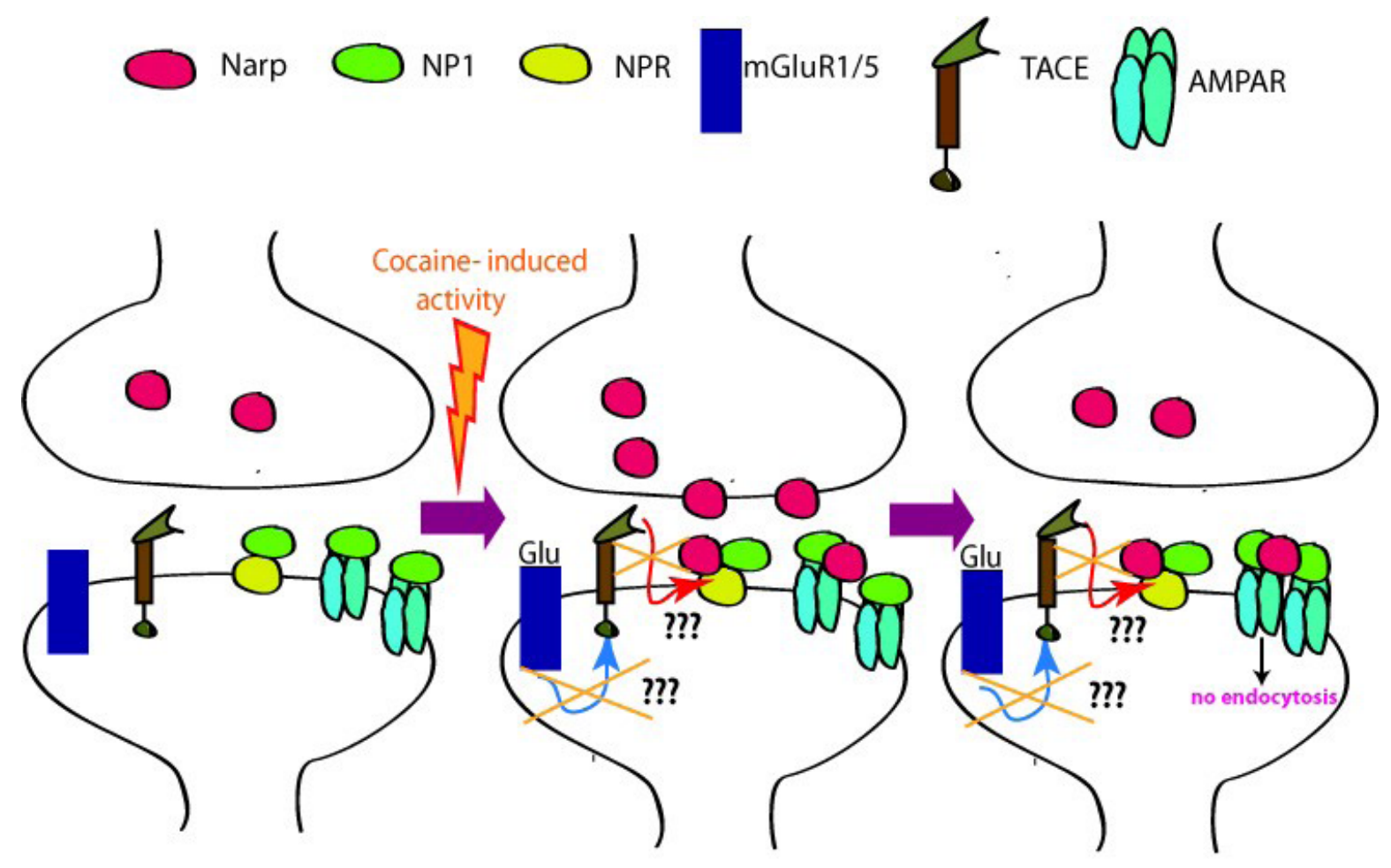

Figure 2. Hypothetical mechanism for the involvement of NPs in cocaine impairment of mGluR5-induced LTD. After cocaine withdrawal, mGluR5-induced LTD is impaired and mGluR5 receptors are down-regulated. This down-regulation may reduce TACE activation and NPR cleavage and release, reducing AMPAR endocytosis what would impair mGluR5-induced LTD.

(i.e. augmented cocaine place preference in both $\mathrm{KO}$ ). Furthermore, we also demonstrated that the expression of GluR1 was decreased only in Narp KO mice withdrawn from cocaine implying that the absence of Narp affects cocaine modulation of AMPAR and therefore increases its behavioral effects. In conclusion, according to the evidence discussed here pentraxins ability to regulate clustering and promote endocytosis of the AMPAR may play a protective role in cocaine induced neuroadaptations.

\section{Acknowledgments}

We would like to thank Armina Wiggins and Khaled Moussawi for helpful comments on the manuscript. This study was supported by United States Public Health Services (Grants DA03906 and DA015851).

\section{References}

1. Kalivas PW, O'Brien C. Drug addiction as a pathology of staged neuroplasticity. Neuropsychopharmacology 2007;33:166-80.
2. Conrad KL, Tseng KY, Uejima JL, et al., Formation of accumbens GluR2-lacking AMPA receptors mediates incubation of cocaine craving. Nature 2008;454:118-121.

3. Boudreau AC, Wolf ME. Behavioral sensitization to cocaine is associated with increased AMPA receptor surface expression in the nucleus accumbens. J Neurosci 2005;25:9144-51.

4. Kourrich S, Rothwell PE, Klug JR, et al. Cocaine experience controls bidirectional synaptic plasticity in the nucleus accumbens. J Neurosci 2007;27:7921-8.

5. Di Ciano P, Everitt BJ. Dissociable effects of antagonism of NMDA and AMPA/KA receptors in the nucleus accumbens core and shell on cocaine-seeking behavior. Neuropsychopharmacology 2001;25:341-60.

6. Pierce RC, Bell K, Duffy P, et al. Repeated cocaine augments excitatory amino acid transmission in the nucleus accumbens only in rats having developed behavioral sensitization. J Neurosci 1996;16:1550-60.

7. Cornish JL, Kalivas PW. Glutamate transmission in the nucleus accumbens mediates relapse in cocaine addiction. J Neurosci 2000;20:89RC.

8. Baker DA, McFarland K, Lake RW, et al. Neuroadaptations in cystine-glutamate exchange underlie cocaine relapse. Nat Neurosci 2003;6:743-9.

9. Moran MM, McFarland K, Melendez RI, et al. Cystine/glutamate exchange regulates metabotropic glutamate receptor presynaptic inhibition of excitatory 
transmission and vulnerability to cocaine seeking. J Neurosci 2005;25:6389-93.

10. McFarland K, Lapish CC, Kalivas PW. Prefrontal glutamate release into the core of the nucleus accumbens mediates cocaine-induced reinstatement of drug-seeking behavior. J Neurosci 2003;23:3531-7.

11. O'Brien R, Xu D, Mi R, et al. Synaptically targeted narp plays an essential role in the aggregation of AMPA receptors at excitatory synapses in cultured spinal neurons. J Neurosci 2002;22:4487-98.

12. O'Brien RJ, Xu D, Petralia RS, et al. Synaptic clustering of AMPA receptors by the extracellular immediate-early gene product Narp. Neuron 1999;23:30923.

13. Kirkpatrick LL, Matzuk MM, Dodds DC, et al. Biochemical interactions of the neuronal pentraxins: neuronal pentraxin (NP) receptor binds to taipoxin and taipoxin-associated calcium-binding protein 49 via NP1 and NP2. J Biol Chem 2000;275:17786-92.

14. Xu, D., Hopf C, Reddy R, et al., Narp and NP1 form heterocomplexes that function in developmental and activity-dependent synaptic plasticity. Neuron 2003;39:513-28.

15. Cho, RW, Park J, Wolff SB, et al. mGluR1/5-dependent long-term depression requires the regulated ectodomain cleavage of neuronal pentraxin NPR by TACE. Neuron 2008;57:858-71.

16. Pacchioni AM, Vallone J, Worley PF, et al. Neuronal pentraxins modulate cocaine-induced neuroadaptations. J Pharmacol Exp Ther 2009;328:183-92.

17. Bachtell, R.K., Choi KH, Simmons DL, et al. Role of GluR1 expression in nucleus accumbens neurons in cocaine sensitization and cocaine-seeking behavior. Eur J Neurosci 2008;27:2229-40.

18. Swanson CJ, Baker DA, Carson D, et al. Repeated cocaine administration attenuates group I metabotropic glutamate receptor-mediated glutamate release and behavioral activation: a potential role for Homer. J Neurosci 2001;21:9043-52.

19. Moussawi K, Pacchioni A, Moran M, et al. NAcetylcysteine reverses cocaine-induced metaplasticity. Nat Neurosci 2009;12:182-9.

20. Baptista MA, Martin-Fardon R, Weiss F. Preferential effects of the metabotropic glutamate $2 / 3$ receptor agonist LY379268 on conditioned reinstatement versus primary reinforcement: comparison between cocaine and a potent conventional reinforcer. J Neurosci 2004;24:4723-7.

21. Peters J, Kalivas PW. The group II metabotropic glutamate receptor agonist, LY379268, inhibits both cocaine- and food-seeking behavior in rats. Psychopharmacology (Berl) 2006;186:143-9. 